\title{
Growing Vortex Rossby Waves with Azimuthal Wavenumber One in Quasigeostrophic System
}

\author{
Takahiro ITO \\ Office of Marine Prediction, Japan Meteorological Agency, Tokyo, Japan \\ Shusuke NISHIMOTO \\ Numerical Prediction Division, Japan Meteorological Agency, Tokyo, Japan \\ and \\ Hirotada KANEHISA \\ Nonaffiliated, 6-19-7, Matsuba, Ryugasaki-shi, Ibaraki, Japan \\ (Manuscript received 6 April 2018, in final form 7 August 2018)
}

\begin{abstract}
In this study, we show analytically that vortex Rossby waves (VRWs) with azimuthal wavenumber $m=1$ in a basic axisymmetric vortex can grow exponentially in a quasi-geostrophic system, although they cannot do so in a barotropic system.

VRWs grow exponentially if Rayleigh's condition and Fjørtoft's condition are satisfied. Satisfying Rayleigh's condition means that two horizontally aligned VRWs at two different radii propagate (here and hereafter "propagate" refers to propagation relative to the fluid) azimuthally counter to each other. Satisfying Fjørtoft's condition means that the cyclonic advective angular velocity of the basic vortex is distributed radially so as to enable the VRWs to be phase-locked with each other. Under these conditions, a strong mutual interaction between the VRWs becomes possible, and thus they grow exponentially.

In a barotropic system, even if Rayleigh's condition is satisfied, the azimuthal counter propagation of VRWs with azimuthal wavenumber $m=1$ is so strong that phase-locking between them cannot occur, and thus they cannot grow exponentially.

In a quasi-geostrophic system, however, the upper and lower VRWs of the first baroclinic vertical mode are equal in magnitude and have opposite signs. Because of this baroclinic structure, the azimuthal counter propagation of the horizontally aligned VRWs is suppressed by the vertical interactions between the upper and lower VRWs. Consequently, horizontally aligned VRWs with azimuthal wavenumber $m=1$ may become phaselocked, and hence they may grow exponentially. By analytically solving the linear problem of VRWs in a quasigeostrophic system, we show that this is indeed the case.
\end{abstract}

Keywords vortex Rossby wave; potential vorticity; instability

Corresponding author: Takahiro Ito, Office of Marine Prediction, Japan Meteorological Agency, 1-3-4, Otemachi, Chiyoda-ku, Tokyo 100-8122, Japan

E-mail: t_ito@met.kishou.go.jp

J-stage Advance Published Date: 7 September 2018

(C) The Author(s) 2018. This is an open access article published by the Meteorological Society of Japan under a Creative Commons Attribution 4.0 International (CC BY 4.0) license (http://creativecommons.org/license/by/4.0). 


\section{Introduction}

Vortex Rossby waves (VRWs) exist where a radial gradient $d Q / d r$ of the basic axisymmetric potential vorticity (PV) $Q$ is present. The VRWs propagate (here and hereafter "propagate" refers to propagation relative to the fluid) azimuthally in the direction to the left of $d Q / d r$. In other words, the propagation is anticyclonic if $d Q / d r<0$, and cyclonic if $d Q / d r>0$. In addition, the VRWs are cyclonically advected by the basic angular velocity $\Omega$ induced by $Q$. If the radial gradient $d Q / d r$ is not sign-definite e.g., if $d Q / d r$ $>0$ at a radius $r^{\prime}$ and $d Q / d r<0$ at another radius $r^{\prime \prime}$ that is not so far from $r^{\prime}$ then two VRWs at $r^{\prime}$ and $r^{\prime \prime}$ propagate azimuthally counter to each other (satisfying Rayleigh's condition). Moreover, if the cyclonic advection by the basic angular velocity $\Omega$ is distributed radially such that the two VRWs at $r^{\prime}$ and $r^{\prime \prime}$ have the same angular phase velocity, that is, they are phaselocked with each other (satisfying Fjørtoft's condition) then they can interact mutually and strongly to amplify each other. Consequently, they grow exponentially in time (Hoskins et al. 1985).

These features of VRW propagation and interaction are now well known, and VRWs are being considered and investigated as one of the main components of asymmetric disturbances in a tropical cyclone. For example, Montgomery and Kallenbach (1997) presented a barotropic theory for the propagation and interaction of VRWs in a basic vortex, and they investigated the effects of the radial propagation and axisymmetrization of VRWs on the basic vortex. Peng, M. S. et al. (2014) and Peng, J. et al. (2014) investigated the radial propagation and axisymmetrization of baroclinic VRWs in a two-layer model. Gao and Zhu (2016) investigated the radial and vertical propagation of baroclinic VRWs on a baroclinic basic vortex. Schubert et al. (1999) considered the polygonal eyewall of a tropical cyclone in terms of VRWs. The annular ring of high PV at the eyewall supports the counter-propagation of VRWs at the inside and outside edges. The inside VRW propagates cyclonically because $d Q / d r>0$, while the outside VRW propagates anticyclonically because $d Q / d r<0$. Because of the high $\mathrm{PV}$ of the annular ring, the cyclonic advective angular velocity $\Omega$ at the outside edge is larger than that at the inside edge. Thus, the two counterpropagating VRWs may satisfy Fjørtoft's condition and be phase-locked with each other to grow exponentially. These studies showed analytically and numerically that the VRWs actually grow to generate the polygonal eyewall. Chen and Yau (2001) analyzed the spiral rain bands of a numerically simulated tropical cyclone and reported that the propagation properties were compatible with VRW theory. Reasor and Montgomery (2001) considered the vortex resiliency of a tropical cyclone in a vertically sheared environmental flow in terms of VRWs. Nishimoto and Kanehisa (2018) analytically investigated the relationship between the vortex resiliency and vertical interaction of the upper and lower VRWs.

According to numerical and observational studies (Wang 2002), asymmetric disturbances in a tropical cyclone are dominated by VRWs with azimuthal wavenumbers $m=1$ and $m=2$. However, as Reznik and Dewar (1994) reported, disturbances with an azimuthal wavenumber $m=1$ cannot grow exponentially in a barotropic linearized system. However, the analytical solution derived by Smith and Rosenbluth (1990) for a linearized barotropic system showed that disturbances with azimuthal wavenumber $m=1$ can grow algebraically as a function of $t^{\frac{1}{2}}$, as $t \rightarrow \infty$, if the basic angular velocity has a maximum at a radius other than the center. Nolan and Montgomery (2000) further investigated algebraic growth. They reported that perturbations growing as, e.g., $t^{\frac{1}{2}}$, are necessarily accompanied by perturbations decaying as, e.g., $t^{-\frac{1}{2}}$.

Additionally, disturbances with azimuthal wavenumber $m=1$ cannot grow exponentially in a typical barotropic, three-discrete-region model, which has an inner region with low $\mathrm{PV}$, an annulus with high $\mathrm{PV}$, and a surrounding environment with zero vorticity (Terwey and Montgomery 2002). However, in a baroclinic model, they can grow as a result of precessional instability (Flierl 1988). Despite the absence of exponential growth, disturbances with azimuthal wavenumber $m=1$ can grow linearly in time in a barotropic, $(N+1)$-discrete-region model, where $N+1 \geq 3$, with a radially piecewise-uniform basic $\mathrm{PV}$, which has an innermost circular region $\left(0=r_{0}<\right.$ $\left.r<r_{1}\right),(N-1)$ intermediate annular regions $\left(r_{j}<r\right.$ $\left.<r_{j+1}, j=1,2, \ldots, N-1\right)$, and an outermost environment $\left(r_{N}<r<r_{N+1}=\infty\right)$ (Ito and Kanehisa 2013). The basic PV $Q$ is discontinuous at the $N$ jump radii $r_{j}, j=1,2, \ldots, N$. Linear growth of disturbances with azimuthal wavenumber $m=1$ occurs when the basic angular velocities at the two jump radii coincide, e.g., $\Omega_{2}=\Omega_{3}$.

The condition $\Omega_{2}=\Omega_{3}$ implies that $\left[\frac{d Q}{d r}\right]_{r=r_{1}}$ and $\left[\frac{d Q}{d r}\right]_{r=r_{2}}$ have opposite signs. Hence, VRWs with the azimuthal wavenumber $m=1$ at $r_{1}$ and $r_{2}$ namely, $\mathrm{VRW}_{1}$ and $\mathrm{VRW}_{2}$ propagate azimuthally counter to 
each other, and therefore satisfy Rayleigh's instability condition. However, the angular propagation velocity of a VRW increases as the azimuthal wavenumber decreases. Consequently, the counter-propagation of $\mathrm{VRW}_{1}$ and $\mathrm{VRW}_{2}$ becomes so strong that they cannot become phase-locked with each other; that is, they cannot satisfy Fjørtoft's instability condition. Therefore, they cannot grow exponentially in a barotropic system i.e., in a system without vertical structure although they can grow linearly in time. Such linear growth was investigated by Ito and Kanehisa (2013); however, they did not discuss the effect of the vertical structure.

The present study focuses on the importance of vertical interactions for the exponential growth of VRWs. In a quasi-geostrophic system, the disturbances may have a baroclinic vertical structure. Particularly in the first baroclinic mode, the upper and lower perturbations are equal in magnitude and opposite in sign. Consequently, the horizontal circulations induced by the upper and lower vorticity perturbations partly cancel each other. Thus, the azimuthal counterpropagation of $\mathrm{VRW}_{1}$ and $\mathrm{VRW}_{2}$ are reduced by the vertical interaction between the upper and lower VRWs, because the propagation of a VRW is caused by the horizontal circulation induced by the PV perturbation produced by the VRW. If the counterpropagation of $\mathrm{VRW}_{1}$ and $\mathrm{VRW}_{2}$ is reduced, they may satisfy Fjørtoft's condition and thus may become phase-locked with each other and grow exponentially. In this paper, we show analytically that this is indeed the case. Specifically, we present an analytical solution to the linearized problem of an axisymmetric basic vortex with a radially piecewise uniform barotropic $\mathrm{PV}$ in a quasi-geostrophic system.

This paper is organized as follows. In Section 2, we derive the governing equation for a PV disturbance linearized about a basic PV. We take the basic PV to be piecewise uniform in $(N+1)$ regions in the radial direction. In Section 3, we present the analytical solution of the governing equation. In Sections 4 and 5 , we demonstrate the existence of an exponentially growing solution for $N=2$ and $N=3$, respectively. In Section 6, we present a proof of the existence of an exponentially growing solution for $N \geq 4$. In Section 7 , we summarize the conclusions drawn from this study.

\section{Governing equation}

We begin with the following quasi-geostrophic potential vorticity equation in the $f$-plane,

$$
\begin{aligned}
& \frac{\partial q}{\partial t}+\frac{1}{r} \frac{\partial \psi}{\partial r} \frac{\partial q}{\partial \theta}-\frac{1}{r} \frac{\partial q}{\partial r} \frac{\partial \psi}{\partial \theta}=0 \\
& q=\left(\frac{1}{r} \frac{\partial}{\partial r} r \frac{\partial}{\partial r}+\frac{1}{r^{2}} \frac{\partial^{2}}{\partial \theta^{2}}+\frac{f^{2}}{N_{0}^{2}} \frac{\partial^{2}}{\partial z^{2}}\right) \psi+f
\end{aligned}
$$

where, $r, \theta, z$, and $t$ are the radial, azimuthal, vertical, and temporal coordinates, respectively. The vertical coordinate $z$ is Hoskins' pseudo-height (Hoskins and Bretherton 1972). The potential vorticity and stream function are denoted by $q$ and $\psi$, respectively. The Coriolis parameter and reference buoyancy frequency, which are denoted by $f$ and $N_{0}$, respectively, are assumed to be constant.

By linearizing about a basic vortex with a barotropic axisymmetric potential vorticity $Q=Q(r)$, Eq. (1) becomes

$$
\begin{aligned}
& \frac{\partial q^{\prime}}{\partial t}+\Omega \frac{\partial q^{\prime}}{\partial \theta}-\frac{1}{r} \frac{d Q}{d r} \frac{\partial \psi^{\prime}}{\partial \theta}=0 \\
& q^{\prime}=\left(\frac{1}{r} \frac{\partial}{\partial r} r \frac{\partial}{\partial r}+\frac{1}{r^{2}} \frac{\partial^{2}}{\partial \theta^{2}}+\frac{f^{2}}{N_{0}^{2}} \frac{\partial^{2}}{\partial z^{2}}\right) \psi^{\prime},
\end{aligned}
$$

where the primes denote perturbations, and $\Omega=\Omega(r)$ is the angular velocity of the basic vortex flow.

We assume that two rigid horizontal boundaries are located at $z=0$ and $z=H$. Because the basic flow is barotropic, the basic potential temperature is horizontally uniform. This implies that the potential temperature is constant at the horizontal boundaries, because vertical motions do not exist there. Therefore, the potential-temperature perturbation disappears at $z=0$ and $z=H$.

We next consider disturbances of the first baroclinic mode in the vertical direction. Because of the hydrostatic approximation, the vertical derivative of the stream-function perturbation $\frac{\partial \psi^{\prime}}{\partial z}$ is proportional to the potential-temperature perturbation, which vanishes at $z=0$ and $z=H$. Hence, the vertical structures of the perturbations in the stream function $\psi^{\prime}$ and the potential vorticity $q^{\prime}$ are proportional to $\cos \left(\frac{\pi z}{H}\right)$.

Moreover, we assume that the disturbances have an azimuthal structure with wavenumber $m=1$; we thus write the perturbations as follows:

$$
\begin{aligned}
& q^{\prime}(r, \theta, z, t)=\operatorname{Re}\left[\hat{q}(r, t) e^{i \theta}\right] \cos \left(\frac{\pi z}{H}\right), \\
& \psi^{\prime}(r, \theta, z, t)=\operatorname{Re}\left[\hat{\psi}(r, t) e^{i \theta}\right] \cos \left(\frac{\pi z}{H}\right),
\end{aligned}
$$




$$
\hat{q}=\left(\frac{1}{r} \frac{\partial}{\partial r} r \frac{\partial}{\partial r}-\frac{1}{r^{2}}-\kappa^{2}\right) \hat{\psi}, \quad \kappa=\frac{f \pi}{N_{0} H} .
$$

In this study, we analyze the structure of a free mode with the first baroclinic, azimuthal wavenumber $m=$ 1 in a barotropic environmental flow; as noted above, Nishimoto and Kanehisa (2018) analyzed a forced mode in a vertically sheared zonal flow.

By substituting (3) and (4) into (2), we obtain the following expression:

$$
\frac{\partial \hat{q}}{\partial t}+i \Omega \hat{q}-i \frac{1}{r} \frac{d Q}{d r} \hat{\psi}=0 .
$$

Using (5), we can express the stream function $\hat{\psi}$ in (6) as a function of $\hat{q}$, with the aid of the Green's function $G\left(r, r^{\prime}\right)$ :

$$
\hat{\psi}(r, t)=\int_{0}^{\infty} d r^{\prime} G\left(r, r^{\prime}\right) \hat{q}\left(r^{\prime}, t\right),
$$

where the Green's function $G\left(r, r^{\prime}\right)$ is the solution of

$$
\left(\frac{1}{r} \frac{d}{d r} r \frac{d}{d r}-\frac{1}{r^{2}}-\kappa^{2}\right) G\left(r, r^{\prime}\right)=\delta\left(r-r^{\prime}\right),
$$

subject to the following boundary conditions:

$$
\lim _{r \rightarrow \infty} G\left(r, r^{\prime}\right)=0 \text { and } \lim _{r \rightarrow 0}\left|G\left(r, r^{\prime}\right)\right|<\infty .
$$

This solution has the form

$$
\begin{aligned}
& G\left(r, r^{\prime}\right)=-r^{\prime} I_{1}\left(\kappa r^{\prime}\right) K_{1}(\kappa r) \text { for } r^{\prime} \leq r, \\
& G\left(r, r^{\prime}\right)=-r^{\prime} I_{1}(\kappa r) K_{1}\left(\kappa r^{\prime}\right) \text { for } r<r^{\prime},
\end{aligned}
$$

where $I_{1}$ is the modified Bessel function of the first kind of order one, and $K_{1}$ is the modified Bessel function of the second kind of order one. By substituting (7) into (6) and rewriting $r \hat{q}=\tilde{q}$, we obtain the following expression:

$$
\begin{aligned}
& \frac{\partial \tilde{q}(r, t)}{\partial t}+i \Omega(r) \tilde{q}(r, t) \\
& -i \frac{d Q}{d r} \int_{0}^{\infty} d r^{\prime} \frac{G\left(r, r^{\prime}\right)}{r^{\prime}} \tilde{q}\left(r^{\prime}, t\right)=0 .
\end{aligned}
$$

To solve the problem analytically, we assume the basic potential vorticity $Q$ to be piecewise uniform in the radial direction, as shown in Fig. 1. This is a generalization of the Rankine vortex model, which has only two regions of uniform potential vorticity. With more than two regions of uniform potential vorticity, the model can represent various TC-like vortices, such

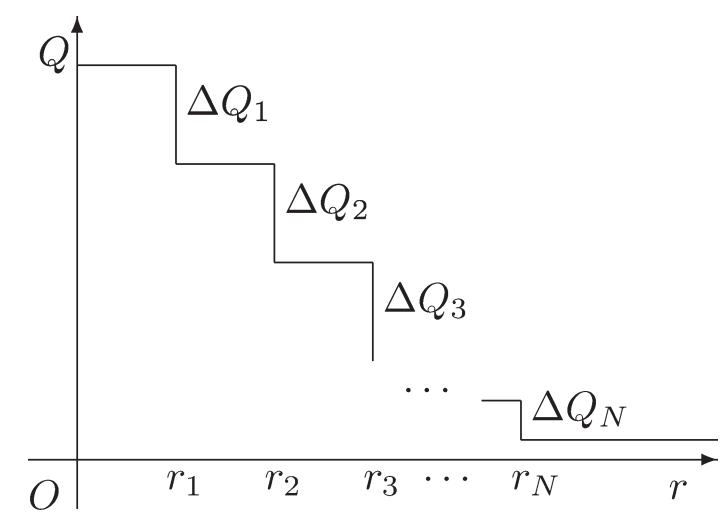

Fig. 1. Radial distribution of the basic potential vorticity.

as a vortex with a moat outside the eyewall.

For this piecewise-uniform distribution, the radial derivative of $Q$ can be expressed in terms of the Dirac delta function:

$$
\frac{d Q}{d r}=-\sum_{j=1}^{N} \Delta Q_{j} \delta\left(r-r_{j}\right) .
$$

The Dirac delta function $\delta\left(r-r_{j}\right)$ is defined by

$$
\delta\left(r-r_{j}\right)=0 \text { if } r \neq r_{j} \text {, and } \int_{0}^{\infty} d r \delta\left(r-r_{j}\right)=1,
$$

and it is the derivative of the step function $\Theta\left(r-r_{j}\right)$ :

$$
\begin{aligned}
& \delta\left(r-r_{j}\right)=\frac{d}{d r} \Theta\left(r-r_{j}\right), \\
& \text { where } \Theta\left(r-r_{j}\right)=\left\{\begin{array}{ll}
1 & \left(r \geq r_{j}\right) \\
0 & \left(r<r_{j}\right)
\end{array}\right. \text {. }
\end{aligned}
$$

For $r \neq r_{j}, j=1,2, \ldots, N$, Eq. (11) reduces to the following:

$$
\frac{\partial \tilde{q}(r, t)}{\partial t}+i \Omega(r) \tilde{q}(r, t)=0 .
$$

The solution is

$$
\tilde{q}(r, t)=\tilde{q}(r, 0) e^{-i \Omega t}
$$

However, this solution disappears if the initial value $\tilde{q}(r, 0)=0$. Hence, for the initial condition $\tilde{q}(r, 0)$ $=0$ for $r \neq r_{j}, j=1,2, \ldots, N$, the potential-vorticity perturbation $\tilde{q}(r, t)$ can be expressed in terms of the Dirac delta function as follows: 


$$
\tilde{q}(r, t)=\sum_{j=1}^{N} \tilde{q}_{j}(t) \delta\left(r-r_{j}\right)
$$

By substituting (12) and (13) into (11), we obtain the following governing equation:

$$
\frac{d \tilde{q}_{j}}{d t}+i \sum_{k=1}^{N} A_{j k} \tilde{q}_{k}=0
$$

where

$$
A_{j k}=\delta_{j k} \Omega_{j}-g_{j k} \Delta Q_{j}, \quad g_{j k}=-\frac{1}{r_{k}} G\left(r_{j}, r_{k}\right),
$$

and

$$
\Omega_{j}=\Omega\left(r_{j}\right)=\frac{1}{2} \sum_{k=j}^{N} \Delta Q_{k}+\frac{1}{2} \sum_{k=1}^{j-1} \frac{r_{k}^{2}}{r_{j}^{2}} \Delta Q_{k} .
$$

While Ito and Kanehisa (2013) used a barotropic system (2-dimensional system), in this study, we use a quasi-geostrophic system (3-dimensional system) in order to consider vertical interactions. By comparing the governing Eq. (14) with that of Ito and Kanehisa (2013), we find that the essential difference is in the form of the Green's function, which is given here by (9) and (10). The difference derives from the term $-\kappa^{2}$ in (8), which represents the influence of the vertical structure. Of course, in the case $\kappa=0$, for which vertical interactions do not exist, the Green's function $\nearrow$

has the same form as that of Ito and Kanehisa (2013). The form of the Green's function given by (9) and (10) makes it possible for VRWs to grow exponentially, as we explain later in this paper.

\section{Analytical solution}

For prescribed initial values of $\tilde{q}_{k}(0)$, the solution to (14) can be written as follows:

$$
\tilde{q}_{j}(t)=\sum_{n=1}^{N} \sum_{k=1}^{N} e^{-i \lambda_{n} t} R_{n j} L_{n k} \tilde{q}_{k}(0)
$$

where $\lambda_{n}, R_{n j}$ and $L_{n j}(n=1,2, \ldots, N)$ are, respectively, the eigenvalues, and the right and left eigenvectors of the coefficient matrix $A$, the components of which are given by $A_{j k}$ in (15). The right and left eigenvectors $R_{n j}$ and $L_{n j}$, respectively, are normalized such that

$$
\sum_{j=1}^{N} L_{n j} R_{m j}=\delta_{n m}, \quad n, m=1,2, \ldots, N .
$$

If an eigenvalue $\lambda$ is non-real, then its complex conjugate $\lambda^{*}$ is also an eigenvalue, because the coefficient matrix $A_{j k}$ is real. The imaginary part of $\lambda$ or $\lambda^{*}$ is positive, which implies that $e^{-i \lambda t}$ or $e^{-i \lambda^{*} t}$ represents exponential growth. Hence, the solution in (17) can grow exponentially if at least one of the eigenvalues $\lambda_{n}, n=1,2, \ldots, N$ is not purely real.

The eigenvalues are the roots of the following eigenvalue equation:

$$
\operatorname{det}(\lambda E-A)=\left|\begin{array}{cccc}
g_{11} \Delta Q_{1}+\lambda-\Omega_{1} & g_{12} \Delta Q_{1} & \ldots & g_{1 N} \Delta Q_{1} \\
g_{21} \Delta Q_{2} & g_{22} \Delta Q_{2}+\lambda-\Omega_{2} & \ldots & g_{2 N} \Delta Q_{2} \\
\vdots & \vdots & \ddots & \vdots \\
g_{N 1} \Delta Q_{N} & g_{N 2} \Delta Q_{N} & \ldots & g_{N N} \Delta Q_{N}+\lambda-\Omega_{N}
\end{array}\right|=0
$$

$\searrow$

where $E$ is the identity matrix. For $\kappa=0$, the Green's function terms become $g_{j k}=\frac{1}{2} \min \left(\frac{r_{j}}{r_{k}}, \frac{r_{k}}{r_{j}}\right)$, and the eigenvalues given by $\lambda_{n}=\Omega_{n+1}$, with $\Omega_{N+1}=0$, are real (see Appendix A). In this case, the solution (17) cannot grow exponentially.

However, if two eigenvalues $\lambda_{n}$ and $\lambda_{m}$ become degenerate, the solution (17) grows as a linear function of time, owing to the resonant interaction between $e^{-i \lambda_{n} t}$ and $e^{-i \lambda_{m} t}$ (Ito and Kanehisa 2013). The term including $e^{-i \lambda_{n} t}$ and $e^{-i \lambda_{m} t}$ of the solution expressed by (17), is expressed as follows: $\propto \frac{e^{-i \lambda_{n} t}-e^{-i \lambda_{m} t}}{\lambda_{n}-\lambda_{m}}$. It be- comes a linear function of time $t$ in the degenerate case $\lambda_{n}=\lambda_{m}$ :

$$
\frac{e^{-i \lambda_{n} t}-e^{-i \lambda_{m} t}}{\lambda_{n}-\lambda_{m}} \rightarrow-i t \quad \text { as } \quad \lambda_{n} \rightarrow \lambda_{m}
$$

For example, if we assume that $\lambda_{1}=\lambda_{2}$, then $\Omega_{2}=$ $\Omega_{3}$, and from (16) it follows that $\Delta Q_{1} r_{1}^{2}+\Delta Q_{2} r_{2}^{2}$ $=0$. Thus, $\Delta Q_{1}$ and $\Delta Q_{2}$ have opposite signs, and Rayleigh's condition is satisfied. This implies that the disturbances at $r=r_{1}$ and $r=r_{2}$ propagate azimuthally counter to each other. These counter propagations are so strong that phase-locking between the distur- 
bances at $r=r_{1}$ and $r=r_{2}$ is impossible. Therefore, exponential growth cannot occur for $\kappa=0$.

However, for $\kappa \gtrsim 0$, counter propagation at $r=r_{1}$ and $r=r_{2}$ is suppressed by the vertical interactions between the upper and lower disturbances of the first baroclinic mode, which have opposite signs. This is because the azimuthal propagation of the upper disturbance and also that of the lower disturbance is caused by horizontal circulations induced by both the upper and lower disturbances.

By the suppression, the propagations appear to be weakened. There is then a possibility that phase-locking between the disturbances at $r=r_{1}$ and $r=r_{2}$ may become possible and that exponential growth may occur. In the following sections, we demonstrate that such exponential growth actually does occur.

\section{The case $N=2$}

In the case $N=2$, the coefficient matrix $A$ becomes as follows:

$$
\begin{aligned}
& A=\left[\begin{array}{cc}
\Omega_{1}-g_{11} \Delta Q_{1} & -g_{12} \Delta Q_{1} \\
-g_{21} \Delta Q_{2} & \Omega_{2}-g_{22} \Delta Q_{2}
\end{array}\right], \\
& \text { with } \Omega_{1}=\frac{\Delta Q_{1}+\Delta Q_{2}}{2} \text { and } \Omega_{2}=\frac{\Delta Q_{1} r_{1}^{2}+\Delta Q_{2} r_{2}^{2}}{2 r_{2}^{2}} \text {. }
\end{aligned}
$$

The eigenvalues $\lambda_{ \pm}$are given by

$$
\begin{aligned}
\lambda_{ \pm}= & \frac{\Delta Q_{1}\left(\frac{1}{2}-g_{11}\right)+\Delta Q_{2}\left(\frac{1}{2}-g_{22}\right)+\Omega_{2} \pm \sqrt{d}}{2} \\
d= & \left\{\Delta Q_{1}\left(\frac{1}{2}-g_{11}-\frac{r_{1}^{2}}{2 r_{2}^{2}}\right)+\Delta Q_{2} g_{22}\right\}^{2} \\
& +4 \Delta Q_{1} \Delta Q_{2} g_{12} g_{21} .
\end{aligned}
$$

If the discriminant $d$ is negative, the eigenvalues $\lambda_{ \pm}$ are not purely real.

In the absence of vertical interactions $(\kappa=0)$, the Green's function terms become $g_{11}=g_{22}=\frac{1}{2}, g_{12}=$ $g_{21}=\frac{r_{1}}{2 r_{2}}$. Therefore, the discriminant $d$ in (21) and the eigenvalues $\lambda_{ \pm}$in (20) become, respectively,

$$
\begin{aligned}
& d=\left(\Delta Q_{1} \frac{r_{1}^{2}}{2 r_{2}^{2}}+\frac{\Delta Q_{2}}{2}\right)^{2}\left(=\Omega_{2}^{2}\right), \\
& \lambda_{ \pm}=\Omega_{2}, \Omega_{3}(=0) .
\end{aligned}
$$

This implies that the discriminant $d$ is non-negative and that the eigenvalues $\lambda_{+}$are definitely real. The solution then cannot grow exponentially, although linear growth is possible in the degenerate case $\Omega_{2}=$ $\Omega_{3}(=0)$.

However, in the presence of vertical interactions $(\kappa$ $\gtrsim 0$ ), the discriminant $d$ in (21) takes the following form:

$$
\begin{aligned}
d=\left[\Delta Q_{1}\left\{\frac{1}{2}-\frac{r_{1}^{2}}{2 r_{2}^{2}}-I_{1}\left(\kappa r_{1}\right) K_{1}\left(\kappa r_{1}\right)\right\}\right. \\
\left.+\Delta Q_{2} I_{1}\left(\kappa r_{2}\right) K_{1}\left(\kappa r_{2}\right)\right]^{2} \\
+4 \Delta Q_{1} \Delta Q_{2}\left\{I_{1}\left(\kappa r_{1}\right) K_{1}\left(\kappa r_{2}\right)\right\}^{2} .
\end{aligned}
$$

Figure 2 shows a comparison between the discriminant $d$ for $\kappa=0$ and that for a fixed $\kappa \gtrsim 0$ under the conditions $\Delta Q_{1}<0$ and $\Delta Q_{2}>0$. For clarity, we show $\operatorname{sgn}(d) \sqrt{|d|}$ instead of $d$. In the presence of vertical interactions $(\kappa \gtrsim 0)$, the discriminant $d$ is negative in the vicinity of $\Delta Q_{2} / \Delta Q_{1}=-r_{1}^{2} / r_{2}^{2}$, which is equivalent to the condition for linear growth in the absence of vertical interactions $(\kappa=0)$; that is, $\Omega_{2}=\Omega_{3}(=0)$. Because $d<0$, the solution can grow exponentially for $\kappa \gtrsim 0$.

Figure 3 shows the amplitude of the solution at $r=$ $r_{1}$ for $\kappa=0$ and that for a fixed $\kappa \gtrsim 0$ as a function of

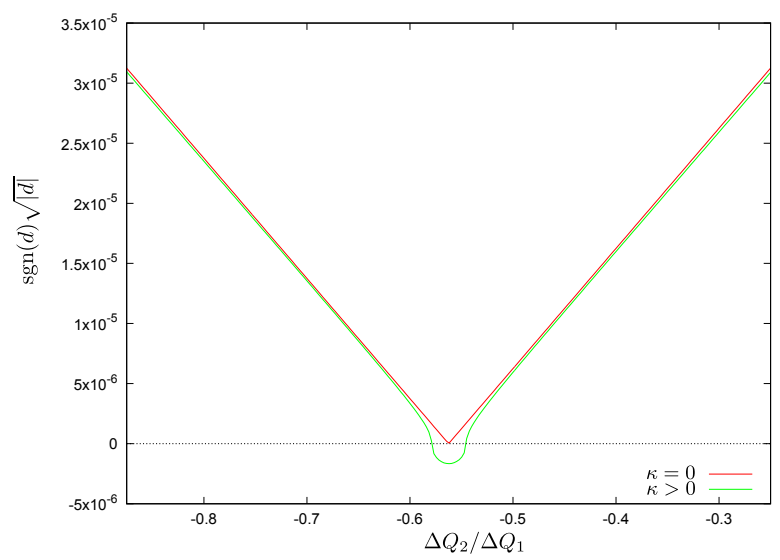

Fig. 2. Graphs of $\operatorname{sgn}(d) \sqrt{|d|}$ for $\kappa=0 \mathrm{~m}^{-1}$ (red) and for $\kappa=2 \times 10^{-6} \mathrm{~m}^{-1}$ (green) as functions of the ratio of the basic potential vorticity jump $\Delta Q_{2} / \Delta Q_{1}$ with $\Delta Q_{1}=-0.002 \mathrm{~s}^{-1}, r_{1}=30 \mathrm{~km}$, and $r_{2}=40 \mathrm{~km}$. In the vicinity of the degenerate intercept $\Delta Q_{2} / \Delta Q_{1}=-r_{1}^{2} / r_{2}^{2}$ of the graph (red) for $\kappa=0$, the value of the graph (green) for $\kappa$ $>0$ becomes negative. 


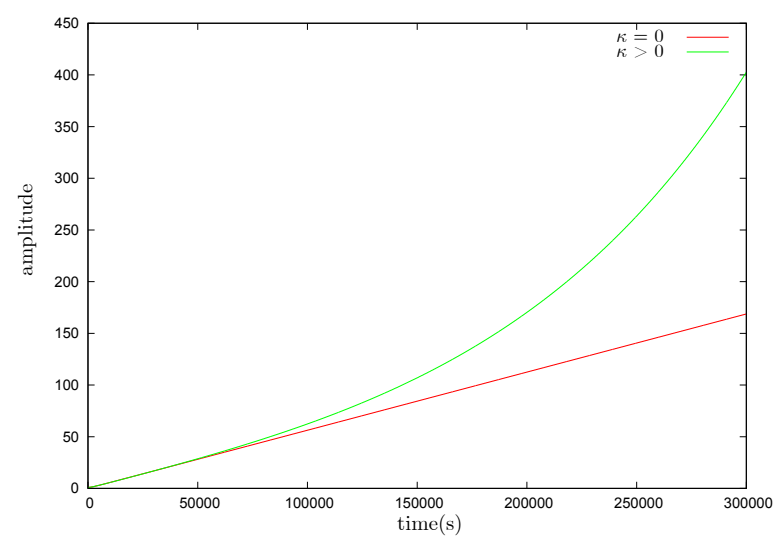

Fig. 3. Amplitude graphs of the solution at $r=r_{1}$ for $\kappa=0 \mathrm{~m}^{-1}$ (red) and for $\kappa=2 \times 10^{-6} \mathrm{~m}^{-1}$ (green) as functions of time $t$. The parameter values are as follows: $r_{1}=30 \mathrm{~km}, r_{2}=40 \mathrm{~km}$, $\Delta Q_{1}=-0.002 \mathrm{~s}^{-1}$, and $\Delta Q_{2}=0.001125 \mathrm{~s}^{-1}$. For these values, the linear growth condition $\Delta Q_{2} / \Delta Q_{1}=-r_{1}^{2} / r_{2}^{2}$ is satisfied for $\kappa=0$.

time $t$ under the condition $\Delta Q_{1} r_{1}^{2}+\Delta Q_{2} r_{2}^{2}=0$, which is equivalent to $\Omega_{2}=\Omega_{3}(=0)$.

In the graph, the red line shows linear growth in the absence of vertical interactions $(\kappa=0)$, while, the green curve represents exponential growth in the presence of vertical interactions $(\kappa \gtrsim 0)$.

\section{The case $N=3$}

We also investigated whether the solution can grow exponentially under the condition $\Omega_{2}=\Omega_{3}$ in the case $N=3$. From (16), the condition is $\Delta Q_{1} r_{1}^{2}+\Delta Q_{2} r_{2}^{2}=$
0 , which is the same as the condition for linear growth when $\kappa=0$ in the case $N=2$.

The eigenvalue equation $D(\lambda, \kappa)=\operatorname{det}(\lambda E-A)=$ 0 is a cubic equation in $\lambda$. Therefore, two of the roots are non-real when the graph of $D(\lambda, \kappa)$ has only one $\lambda$-intercept.

Figure 4 shows the graphs of $\operatorname{sgn}(D)|D|^{\frac{1}{3}}$ instead of $D$ for $\kappa=0$ and for a fixed $\kappa \gtrsim 0$ under the condition of $\Omega_{2}=\Omega_{3}$.

In the absence of vertical interactions $(\kappa=0)$, the graph has two $\lambda$-intercepts. Hence, the eigenvalue equation has three real roots, two of which are degenerate.

On the other hand, in the presence of vertical interactions $(\kappa \gtrsim 0)$, the degenerate $\lambda$-intercept of the graph for $\kappa=0$ disappears, and the graph has only one $\lambda$-intercept. Hence, the eigenvalue equation has non-real roots. The existence of non-real roots implies that, in the presence of vertical interactions $(\kappa \gtrsim 0)$, the solution can also grow exponentially in the case $N$ $=3$.

We next consider the other degenerate conditions, $\Omega_{2}=\Omega_{4}(=0)$ and $\Omega_{3}=\Omega_{4}(=0)$, under which linear growth also occurs in the absence of vertical interactions $(\kappa=0)$.

Figure 5 shows a comparison between the discriminant $d$ (see Appendix B) for $\kappa=0$ with the discriminant for a fixed $\kappa \gtrsim 0$. For clarity, we again plot $\operatorname{sgn}(d)|d|^{\frac{1}{6}}$ instead of $d$. In the presence of vertical interactions $(\kappa \gtrsim 0)$, the discriminant $d$ is negative in the vicinity of $\Omega_{2}=\Omega_{3}$ and $\Omega_{3}=\Omega_{4}(=0)$, which are the linear growth conditions in the absence of vertical interactions $(\kappa=0)$. Because $d<0$, the solution can grow exponentially. However, the discriminant $d$ is
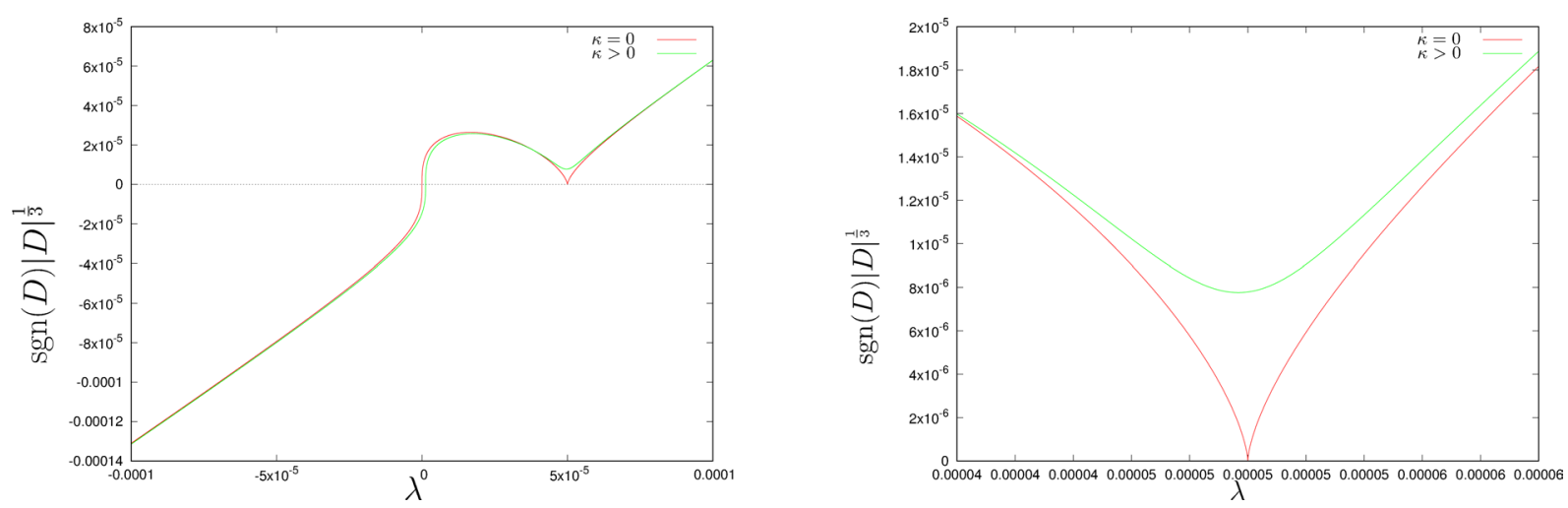

Fig. 4. Graphs of $\operatorname{sgn}(D)|D|^{\frac{1}{3}}$ for $\kappa=0 \mathrm{~m}^{-1}$ (red) and $\kappa=2 \times 10^{-6} \mathrm{~m}^{-1}$ (green) as functions of $\lambda$. (An enlarged view in the vicinity of $\lambda=\Omega_{2}=\Omega_{3}$ is shown at the right) The parameter values are set such that the linear growth condition $\Delta Q_{2} / \Delta Q_{1}=-r_{1}^{2} / r_{2}^{2}$ is satisfied for $\kappa=0$. 


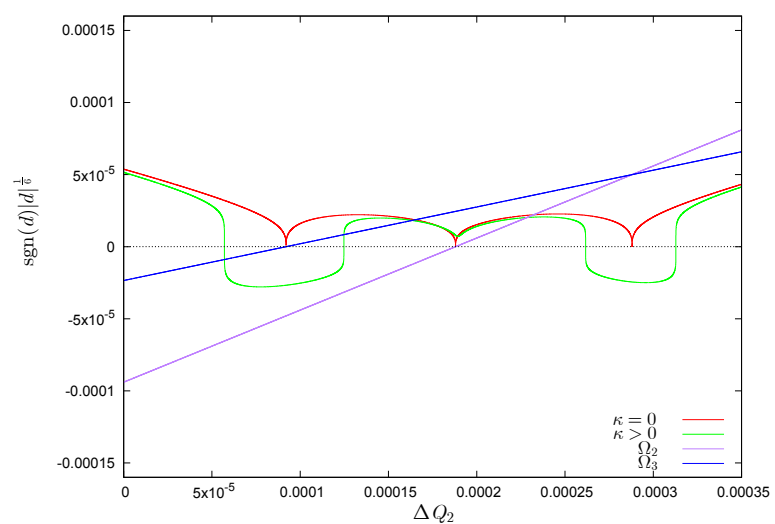

Fig. 5. Graphs of $\operatorname{sgn}(d)|d|^{\frac{1}{6}}$ for $\kappa=0 \mathrm{~m}^{-1}$ (red) and $\kappa=2 \times 10^{-6} \mathrm{~m}^{-1}$ (green) as functions of $\Delta Q_{2}$ with $\Delta Q_{1}=-0.0008 \mathrm{~s}^{-1}, \Delta Q_{3}=0.0001 \mathrm{~s}^{-1}$, $r_{1}=30 \mathrm{~km}, r_{2}=50 \mathrm{~km}$, and $r_{3}=70 \mathrm{~km}$. The slanting lines show $\Omega_{2}$ (purple) and $\Omega_{3}$ (blue). In the vicinities of the degenerate intercepts at $\Omega_{2}=\Omega_{3}$ and $\Omega_{3}=\Omega_{4}(=0)$ of the graph (red) for $\kappa=0$, the values of the graph (green) for $\kappa$ $\gtrsim 0$ become negative, while in the vicinity of $\Omega_{2}=\Omega_{4}(=0)$, the values become positive. positive in the vicinity of $\Omega_{2}=\Omega_{4}(=0)$ and in the presence of vertical interactions $(\kappa \gtrsim 0)$. This implies that the presence of vertical interactions does not always enable the linearly growing solutions to grow exponentially. An example of exponential growth is shown in Fig. 6.

\section{The case of general $N$}

Finally, we investigate whether the solution for general $N \geq 4$ can grow exponentially under the condition $\Omega_{2}=\Omega_{3}$, which is the linear growth condition in the absence of vertical interactions $(\kappa=0)$.

In the same manner as for $N=3$, we examine the graph of $D(\lambda, \kappa)=\operatorname{det}(\lambda E-A)$. From (16) the condition $\Omega_{2}=\Omega_{3}$ means $\Delta Q_{1} r_{1}^{2}+\Delta Q_{2} r_{2}^{2}=0$, so that $\Delta Q_{1}$ and $\Delta Q_{2}$ have opposite signs. We assume that

$$
\Delta Q_{1}<0 \text { and } \Delta Q_{2}, \Delta Q_{3}, \Delta Q_{4}, \ldots, \Delta Q_{N}>0
$$

Under the condition $\Delta Q_{1} r_{1}^{2}+\Delta Q_{2} r_{2}^{2}=0$, Eq. (16) can be rewritten as follows:

$$
\Omega_{n}=\Omega_{2}-\frac{1}{2} \sum_{k=3}^{n-1}\left(1-\frac{r_{k}^{2}}{r_{n}^{2}}\right) \Delta Q_{k} \quad(n=3,4, \ldots, N),
$$

where the basic angular velocities satisfy the follow-

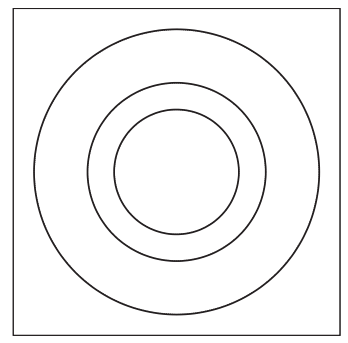

$t=0 \mathrm{~s}$

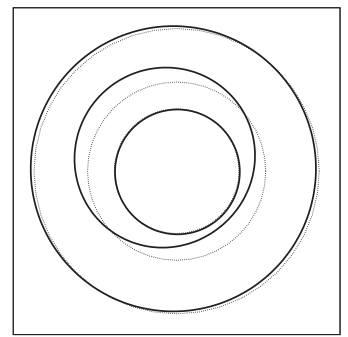

$t=120000 \mathrm{~s}$

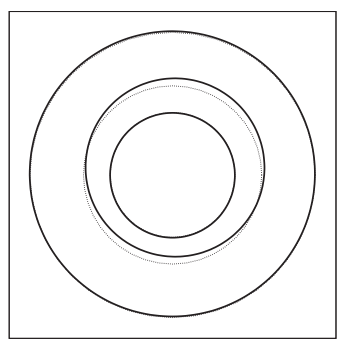

$t=30000 \mathrm{~s}$

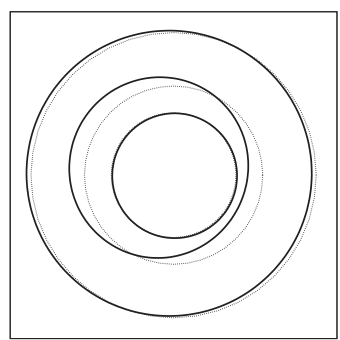

$t=150000 \mathrm{~s}$

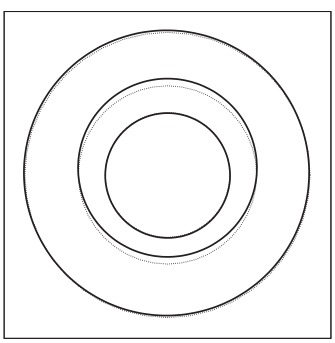

$t=60000 \mathrm{~s}$

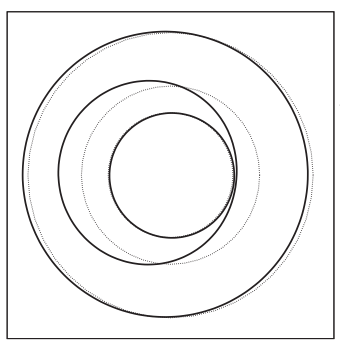

$t=180000 \mathrm{~s}$

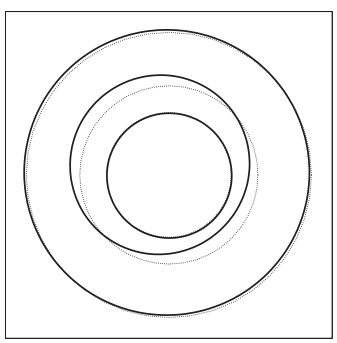

$t=90000 \mathrm{~s}$

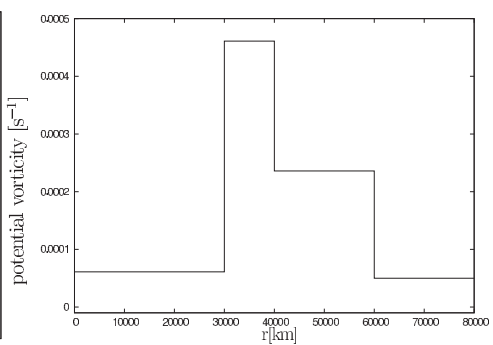

Fig. 6. Example of exponential growth for $\kappa=2 \times 10^{-6} \mathrm{~m}^{-1}$. The lower rightmost panel shows the radial distribution of the basic potential vorticity. In the other panels, the dotted (solid) curves are the iso-PV curves of the basic (total) potential vorticity. The parameter values are as follows: $r_{1}=30 \mathrm{~km}, r_{2}=40 \mathrm{~km}, r_{3}=60 \mathrm{~km}, \Delta Q_{1}$ $=-0.0004 \mathrm{~s}^{-1}, \Delta Q_{2}=0.000225 \mathrm{~s}^{-1}$, and $\Delta Q_{3}=0.000186 \mathrm{~s}^{-1}$. For these values, the linear growth condition (for $\kappa=0$ ) and the exponential growth condition (for $\kappa \gtrsim 0$ ) are satisfied. 
ing equation:

$$
\Omega_{2}=\Omega_{3}>\Omega_{4}>\cdots>\Omega_{N+1}=0 .
$$

The basic angular velocities $\Omega_{n}(n=2,3,4, \ldots, N)$ are the roots of $D(\lambda, 0)=0$, which are the $\lambda$-intercepts of the graph $D(\lambda, 0)$. Additionally, the degenerate root $\tilde{\lambda}=\Omega_{2}=\Omega_{3}$ is the largest eigenvalue in the absence of vertical interactions $(\kappa=0)$. Because $\lim _{\lambda \rightarrow \infty} D(\lambda, \kappa)$ $=+\infty$, the graph is downward convex at the largest degenerate $\lambda$-intercept of $\lambda=\tilde{\lambda}$. Therefore, if the graph leaves the $\lambda$-axis upward at $\lambda=\tilde{\lambda}$ as $\kappa$ increases from zero, the degenerate eigenvalue $\tilde{\lambda}$ becomes two non-real eigenvalues, which are complex conjugates of each other.

The first derivative of $D(\tilde{\lambda}, \kappa)$ with respect to $\kappa$ at $\kappa=0$ is given by (see Appendix C)

$$
\left[\frac{\partial D(\tilde{\lambda}, \kappa)}{\partial \kappa}\right]_{\kappa=0}=0,
$$

while the second derivative is expressed as follows (see Appendix D):

$$
\begin{aligned}
& {\left[\frac{\partial^{2} D(\tilde{\lambda}, \kappa)}{\partial \kappa^{2}}\right]_{\kappa=0}} \\
& =\frac{\Delta Q_{1} \Delta Q_{2}}{8} r_{1}^{2}\left(1-\frac{r_{2}^{2}}{r_{3}^{2}}\right) \\
& \quad \times\left\{1-\frac{r_{1}^{2}}{r_{2}^{2}}+2 \log \left(\frac{r_{1}}{r_{2}}\right)\right\} \prod_{j=4}^{N+1}\left(\Omega_{2}-\Omega_{j}\right) .
\end{aligned}
$$

By substituting (25), (26) and $1-\frac{r_{1}^{2}}{r_{2}^{2}}+2 \log \left(\frac{r_{1}}{r_{2}}\right)<0$ into (28), we obtain the following equation:

$$
\left[\frac{\partial^{2} D(\tilde{\lambda}, \kappa)}{\partial \kappa^{2}}\right]_{\kappa=0}>0 .
$$

Hence, the graph of $D(\lambda, \kappa)$ leaves the $\lambda$-axis upward at $\lambda=\tilde{\lambda}$ as $\kappa$ increases from zero. This implies that vertical interactions $(\kappa>0)$ enable the linearly growing solution to grow exponentially for any $N \geq 2$.

\section{Conclusion}

In this paper, we investigated the growth mechanism of vortex Rossby waves (VRWs) with azimuthal wavenumber $m=1$ in a quasi-geostrophic system. We solved the equation for the potential vorticity (PV) disturbance by linearizing about an axisymmetric basic PV. We assumed the vertical structure of the disturbance to be in the first baroclinic mode. The verti- cal interaction between the VRWs is represented by the non-negative parameter $\kappa=\frac{f \pi}{N_{0} H}$, where $f$ is the Coriolis parameter, $H$ is the fluid depth, and $N_{0}$ is the reference buoyancy frequency. The larger the value of $\kappa$, the stronger is the vertical interaction. To solve the problem analytically, we assumed the axisymmetric basic PV to be piecewise uniform in the radial direction, with $\Delta Q_{j}$ jumps at $r_{j}\left(j=1,2, \ldots, N\right.$, and $0=r_{0}$ $\left.<r_{1}<r_{2}<\cdots<r_{N}<r_{N+1}=\infty\right)$. The solution of the linear problem can then be described as a superposition of the $N$ radial modes, for which the timedependence is expressed as $e^{-i \lambda_{n} t}(n=1,2, \ldots, N)$. Here the $\lambda_{n}$ represent the eigenvalues of the linear problem, which are the roots of the eigenvalue equation $D(\lambda, \kappa)=0$ of degree $N$ in $\lambda$. The polynomial $D(\lambda, \kappa)$ of degree $N$ in $\lambda$ is defined such that $\lim _{\lambda \rightarrow \infty} D(\lambda, \kappa)=\infty$. The solution can grow exponentially if there exists at least one complex conjugate pair of eigenvalues.

In the absence of vertical interactions $(\kappa=0)$, the eigenvalues are $\lambda_{n}=\Omega_{n+1}(n=1,2, \ldots, N)$, where $\Omega_{n+1}$ is the basic angular velocity at $r_{n+1}(n=1,2, \ldots$, $N-1)$ and $\Omega_{N+1}=0$ is the basic angular velocity at $r_{N+1}=\infty$. Because the eigenvalues are real, exponential growth is impossible in the absence of vertical interactions. Although the solution cannot grow exponentially, linear growth is still possible in the degenerate case $\tilde{\lambda}=\lambda_{j}=\lambda_{k}$ for some $j \neq k$; that is, $D(\tilde{\lambda}, 0)=0$ and $\left[\frac{\partial D(\lambda, 0)}{\partial \lambda}\right]_{\lambda=\tilde{\lambda}}=0$ (Ito and Kanehisa 2013).

In this degenerate case, the two VRWs, which are aligned horizontally at different radii, amplify each other linearly and propagate in directions azimuthally counter to each other. That is, Rayleigh's condition is satisfied. However, their azimuthal counter-propagation is so strong that they cannot be phase-locked to each other. That is, Fjørtoft's condition is not satisfied.

On the other hand, in the presence of vertical interactions $(\kappa \gtrsim 0)$, the azimuthal counter-propagation is suppressed by the vertical interactions between the upper and lower VRWs, because the upper and lower PV perturbations of the first baroclinic mode are equal in magnitude and have opposite signs. Specifically, the cyclonic horizontal circulation around the lower (upper) positive PV perturbation and the anticyclonic horizontal circulation around the upper (lower) negative PV perturbation partially cancel each other out. The partial cancelation implies the suppression of VRW propagation because the propagation of a VRW is caused by circulation induced by the PV per- 
turbation. Owing to the suppression of the azimuthal counter-propagation, the two horizontally aligned VRWs may be phase-locked to each other and may therefore grow exponentially.

In Sections 4, 5, and 6, we presented the following results analytically for $N \geq 2$. In the absence of vertical interactions $(\kappa=0)$, the eigenvalues are $\lambda_{n}=\Omega_{n+1}$ $(n=1,2, \ldots, N-1)$ and $\lambda_{N}=\Omega_{N+1}=0$; they are the $N$ roots of the eigenvalue equation $D(\lambda, 0)=0$ of degree $N$. In the degenerate case $\tilde{\lambda}=\lambda_{1}=\lambda_{2}>0$, which implies that $\Omega_{2}=\Omega_{3}$, the graph of $D(\lambda, 0)$ has one degenerate $\lambda$-intercept at $\tilde{\lambda}$; that is, $D(\tilde{\lambda}, 0)=0$, $\left[\frac{\partial D(\lambda, 0)}{\partial \lambda}\right]_{\lambda=\tilde{\lambda}}=0$ and $\left[\frac{\partial^{2} D(\lambda, 0)}{\partial \lambda^{2}}\right]_{\lambda=\tilde{\lambda}}>0$. Then, the solution grows as a linear function of time $t$. The first and second derivatives of $D(\tilde{\lambda}, \kappa)$ with respect to $\kappa$ at $\kappa=0$ become $\left[\frac{\partial D(\tilde{\lambda}, \kappa)}{\partial \kappa}\right]_{\kappa=0}=0,\left[\frac{\partial^{2} D(\tilde{\lambda}, \kappa)}{\partial \kappa^{2}}\right]_{\kappa=0}>0$, respectively. The above equality and inequality imply that the graph of $D(\lambda, 0)$ leaves the $\lambda$-axis upward at the degenerate $\tilde{\lambda}$ as $\kappa$ increases from zero. In other words, they imply that the degenerate root $\tilde{\lambda}$ becomes one complex conjugate pair. Consequently, the solution grows as an exponential function of time $t$.

For $N=3$, another degenerate case where $\Omega_{2}=\Omega_{4}$ $(=0)$ is also possible in the absence of vertical interactions $(\kappa=0)$. Of course, in this case, the solution grows as a linear function of time $t$. However, in the presence of vertical interactions $(\kappa \gtrsim 0)$, the degenerate $\lambda$-intercept at $\tilde{\lambda}$ (corresponding to $\Omega_{2}=\Omega_{4}$ ) of the $D(\lambda, 0)$ graph becomes two distinct $\lambda$-intercepts instead of disappearing. Consequently, the linearly growing solution becomes a non-growing solution. Hence, the vertical interactions $(\kappa \gtrsim 0)$ do not always enable a linearly growing solution to grow exponentially. In future work, we will investigate under what conditions the vertical interactions $(\kappa \gtrsim 0)$ enable a degenerate, linearly growing solution to grow exponentially.

When the vertical interaction is so strong that the horizontal circulations around the upper and lower PV perturbations totally cancel each other out, then, the VRWs do not propagate but are simply advected by the basic angular velocity at each radius. This simple advection implies that the solution does not grow either linearly or exponentially. That is, an excessively strong vertical interaction (excessively large $\kappa$ ) will stabilize the solution. In future work, we also plan to investigate in what range of $\kappa$ a degenerate, linearly growing solution becomes an exponentially growing solution.

\section{Acknowledgments}

The authors would like to thank the two anonymous reviewers for their many valuable comments and suggestions, which substantially improved the manuscript.

\section{Appendix A: Eigenvalues in the absence of vertical interactions $(\kappa=0)$}

In the absence of vertical interactions $(\kappa=0)$, the eigenvalue equation $D(\lambda, \kappa)=\operatorname{det}(\lambda E-A)=0$ is as follows:

$$
D(\lambda, 0)=\left|\begin{array}{cccc}
\frac{\Delta Q_{1}}{2}+\lambda-\Omega_{1} & \frac{r_{1}}{2 r_{2}} \Delta Q_{1} & \ldots & \frac{r_{1}}{2 r_{N}} \Delta Q_{1} \\
\frac{r_{1}}{2 r_{2}} \Delta Q_{2} & \frac{\Delta Q_{2}}{2}+\lambda-\Omega_{2} & \ldots & \frac{r_{2}}{2 r_{N}} \Delta Q_{2} \\
\vdots & \vdots & \ddots & \vdots \\
\frac{r_{1}}{2 r_{N}} \Delta Q_{N} & \frac{r_{2}}{2 r_{N}} \Delta Q_{N} & \ldots & \frac{\Delta Q_{N}}{2}+\lambda-\Omega_{N}
\end{array}\right|=0
$$

We add the second column $\times\left(-\frac{r_{1}}{r_{2}}\right)$ to the first column, so that the $(j, 1)$ components for $j=3,4, \ldots, N$ disappear. Moreover, by substituting $\Omega_{1}=\Omega_{2}+\frac{\Delta Q_{1}}{2}\left(1-\frac{r_{1}^{2}}{r_{2}^{2}}\right)$, the determinant $D(\lambda, 0)$ in (30) becomes 


$$
D(\lambda, 0)=\left(\lambda-\Omega_{2}\right)\left|\begin{array}{ccccc}
1 & \frac{r_{1}}{2 r_{2}} \Delta Q_{1} & \frac{r_{1}}{2 r_{3}} \Delta Q_{1} & \ldots & \frac{r_{1}}{2 r_{N}} \Delta Q_{1} \\
-\frac{r_{1}}{r_{2}} & \frac{1}{2} \Delta Q_{2}+\left(\lambda-\Omega_{2}\right) & \frac{r_{2}}{2 r_{3}} \Delta Q_{2} & \ldots & \frac{r_{2}}{2 r_{N}} \Delta Q_{2} \\
0 & \frac{r_{2}}{2 r_{3}} \Delta Q_{3} & \frac{1}{2} \Delta Q_{3}+\left(\lambda-\Omega_{3}\right) & \ldots & \frac{r_{3}}{2 r_{N}} \Delta Q_{3} \\
\vdots & \vdots & \vdots & \ddots & \vdots \\
0 & \frac{r_{2}}{2 r_{N}} \Delta Q_{N} & \frac{r_{3}}{2 r_{N}} \Delta Q_{N} & \ldots & \frac{1}{2} \Delta Q_{N}+\left(\lambda-\Omega_{N}\right)
\end{array}\right|
$$

Then we add the first column $\times\left(-\frac{r_{1}}{2 r_{j}} \Delta Q_{1}\right)$ to the $j$-th column, so that the $(1, j)$ components for $j=2,3, \ldots, N$ disappear. We then add the first row $\times \frac{r_{1}}{r_{2}}$ to the second row so that the $(2,1)$ component disappears:

$$
D(\lambda, 0)=\left(\lambda-\Omega_{2}\right)\left|\begin{array}{ccccc}
1 & 0 & 0 & \cdots & 0 \\
0 & \frac{1}{2} \Delta Q_{2}+\frac{r_{1}^{2}}{2 r_{2}^{2}} \Delta Q_{1}+\left(\lambda-\Omega_{2}\right) & \frac{r_{2}}{2 r_{3}} \Delta Q_{2}+\frac{r_{1}^{2}}{2 r_{2} r_{3}} \Delta Q_{1} & \ldots & \frac{r_{2}}{2 r_{N}} \Delta Q_{2}+\frac{r_{1}^{2}}{2 r_{2} r_{N}} \Delta Q_{1} \\
0 & \frac{r_{2}}{2 r_{3}} \Delta Q_{3} & \frac{1}{2} \Delta Q_{3}+\left(\lambda-\Omega_{3}\right) & \ldots & \frac{r_{3}}{2 r_{N}} \Delta Q_{3} \\
\vdots & \vdots & \vdots & \ddots & \vdots \\
0 & \frac{r_{2}}{2 r_{N}} \Delta Q_{N} & \frac{r_{3}}{2 r_{N}} \Delta Q_{N} & \cdots & \frac{1}{2} \Delta Q_{N}+\left(\lambda-\Omega_{N}\right)
\end{array}\right| .
$$

In the same way, by substituting $\Omega_{n}=\Omega_{n+1}+\frac{1}{2}\left(\frac{1}{r_{n}^{2}}-\frac{1}{r_{n+1}^{2}}\right) \sum_{k=1}^{n} r_{k}^{2} \Delta Q_{k}(n=2,3, \ldots, N-1), \Omega_{N}=\sum_{k=1}^{N-1} \frac{r_{k}^{2}}{2 r_{N}^{2}} \Delta Q_{k}+\frac{\Delta Q_{N}}{2}$ and performing elementary matrix operations, the determinant $D(\lambda, 0)$ in $(30)$ becomes

$$
D(\lambda, 0)=\left(\lambda-\Omega_{2}\right)\left(\lambda-\Omega_{3}\right) \cdots\left(\lambda-\Omega_{N}\right)\left(\lambda-\Omega_{N+1}\right),
$$

where $\Omega_{N+1}=0$. Hence, in the absence of vertical interactions ( $\left.\kappa=0\right)$, the eigenvalues are given by $\lambda=\Omega_{2}, \Omega_{3}, \ldots$, $\Omega_{N+1}$.

\section{Appendix B: Discriminant of a cubic equation}

We consider a cubic equation with real coefficients (the coefficient of $x^{3}$ can be assumed to be unity without loss of generality):

$$
x^{3}+a x^{2}+b x+c=0 .
$$

The roots of (32) are denoted by $x=\alpha, \beta, \gamma$. Let us consider the following expression:

$$
d=(\alpha-\beta)^{2}(\beta-\gamma)^{2}(\gamma-\alpha)^{2} .
$$

If $\alpha, \beta$, and $\gamma$ are all real, then $d$ is positive. If two of the roots are equal to each other, then, $d$ disappears. If $\alpha, \beta$, or $\gamma$ is non-real, then its complex conjugate will also be a root of (32) because the coefficients of (32) are real. Let the non-real roots be $A \pm B i$, and $C$ with real $A, B$, and $C$. Then, $d=-4 B^{2}\left\{(A-C)^{2}+B^{2}\right\}^{2}$. Therefore, $d$ has the following algebraic properties:

$$
d \begin{cases}>0 \Rightarrow & \begin{array}{l}
\text { three distinct real roots, } \\
\text { two distinct real roots (double root } \\
\text { case) or one real root (triple root case) },
\end{array} \\
<0 \Rightarrow \begin{array}{l}
\text { one real root and two distinct complex } \\
\text { roots. }
\end{array}\end{cases}
$$

From the relationship between the sums and products of the roots and coefficients (Vieta's formula), the discriminant $d$ can be expressed as follows:

$$
d=-4 b^{3}-27 c^{2}+a^{2} b^{2}+18 a b c-4 a^{3} c .
$$

The order of $d$ is equal to that of $x^{6}$. 
Appendix C: Proof of $\left[\frac{\partial D(\tilde{\lambda}, \kappa)}{\partial \kappa}\right]_{\kappa=0}=0$

Let $M=\tilde{\lambda} E-A$. From (15), the components $M_{j k}$ of $M$ are as follows:

$$
M_{j k}=\left(\tilde{\lambda}-\Omega_{j}\right) \delta_{j k}+g_{j k} \Delta Q_{j}(j, k=1,2, \ldots, N) .
$$

In terms of $M_{j k}$, the determinant $D=D(\tilde{\lambda}, \kappa)=$ $\operatorname{det}(\tilde{\lambda} E-A)$ can be expressed as

$$
D=\frac{1}{N !} \epsilon_{j_{1} j_{2} \cdots j_{N}} \epsilon_{k_{1} k_{2} \cdots k_{N}} M_{j_{1} k_{1}} M_{j_{2} k_{2}} \cdots M_{j_{N} k_{N}}
$$

where $\epsilon_{j_{1} j_{2} \cdots j_{N}}$ and $\epsilon_{k_{1} k_{2} \cdots k_{N}}$ are the following permutation symbols:

$$
\epsilon_{j_{1} j_{2} \cdots j_{N}}=\left\{\begin{array}{l}
+1\left\{\begin{array}{l}
\text { if }\left(j_{1}, j_{2}, \ldots, j_{N}\right) \text { is an even } \\
\text { permutation of }(1,2, \ldots, N),
\end{array}\right. \\
-1\left\{\begin{array}{r}
\text { if }\left(j_{1}, j_{2}, \ldots, j_{N}\right) \text { is an odd } \\
\text { permutation of }(1,2, \ldots, N), \\
\text { otherwise },
\end{array}\right.
\end{array}\right.
$$

and the following summation symbols are omitted:

$$
\sum_{j_{1}=1}^{N} \sum_{j_{2}=1}^{N} \cdots \sum_{j_{N}=1}^{N} \sum_{k_{1}=1}^{N} \sum_{k_{2}=1}^{N} \cdots \sum_{k_{N}=1}^{N}
$$

Then the partial derivative of the determinant $D=$ $D(\tilde{\lambda}, \kappa)=\operatorname{det}(\tilde{\lambda} E-A)$ with respect to $\kappa$ becomes

$$
\dot{D}=\frac{1}{(N-1) !} \epsilon_{j_{1} j_{2} \cdots j_{N}} \epsilon_{k_{1} k_{2} \cdots k_{N}} \dot{M}_{j_{1} k_{1}} M_{j_{2} k_{2}} \cdots M_{j_{N} k_{N}}
$$

where $\left({ }^{\circ}\right)$ denotes the partial derivative of () with respect to $\kappa$. By substituting (15), we obtain the partial derivatives of the components $\dot{M}_{j k}$ :

$$
\dot{M}_{j k}=\frac{\partial}{\partial \kappa}\left\{\left(\tilde{\lambda}-\Omega_{j}\right) \delta_{j k}+g_{j k} \Delta Q_{j}\right\}=\dot{g}_{j k} \Delta Q_{j} .
$$

Moreover, using (9), (10), and (15) yields the following relationships:

$$
\dot{g}_{j k}= \begin{cases}\frac{d}{d \kappa}\left\{I_{1}\left(\kappa r_{j}\right) K_{1}\left(\kappa r_{k}\right)\right\} & \text { for } r_{j}<r_{k} \\ \frac{d}{d \kappa}\left\{I_{1}\left(\kappa r_{k}\right) K_{1}\left(\kappa r_{j}\right)\right\} & \text { for } r_{k}<r_{j}\end{cases}
$$

First, we consider the case $r_{j}<r_{k}$. The modified Bessel functions $I_{n}(x)$ and $K_{n}(x)$ are defined as follows:

$$
\begin{aligned}
I_{n}(x)= & \sum_{k=0}^{\infty} \frac{1}{k ! \Gamma(k+n+1)}\left(\frac{x}{2}\right)^{2 k+n}, \\
K_{n}(x)= & \frac{1}{2} \sum_{k=0}^{n-1} \frac{(-1)^{k}(n-k-1) !}{k !}\left(\frac{x}{2}\right)^{2 k-n} \\
& +(-1)^{n+1} \sum_{k=0}^{\infty} \frac{1}{k !(n+k) !}\left(\frac{x}{2}\right)^{2 k+n} \\
& \times\left\{\log \left(\frac{x}{2}\right)-\frac{1}{2} \Psi(k+1)-\frac{1}{2} \Psi(k+n+1)\right\},
\end{aligned}
$$

where $\Psi$ is the digamma function. By using the following formulas for the modified Bessel functions,

$$
\begin{aligned}
& I_{n}^{\prime}(x)=\frac{I_{n-1}(x)+I_{n+1}(x)}{2}, \\
& I_{n}^{\prime}(x) K_{n}(x)-I_{n}(x) K_{n}^{\prime}(x)=\frac{1}{x},
\end{aligned}
$$

we can express the derivative $\dot{g}_{j k}$ for $r_{j}<r_{k}$ in (39) in terms of the primitive modified Bessel functions, as follows:

$$
\begin{aligned}
\dot{g}_{j k}= & r_{j} \frac{I_{0}\left(\kappa r_{j}\right)+I_{2}\left(\kappa r_{j}\right)}{2} K_{1}\left(\kappa r_{k}\right) \\
& +r_{k} I_{1}\left(\kappa r_{j}\right) \frac{1}{I_{1}\left(\kappa r_{k}\right)} \\
& \times\left\{\frac{I_{0}\left(\kappa r_{k}\right)+I_{2}\left(\kappa r_{k}\right)}{2} K_{1}\left(\kappa r_{k}\right)-\frac{1}{\kappa r_{k}}\right\} .
\end{aligned}
$$

To calculate $\lim _{\kappa \rightarrow 0} \dot{g}_{j k}$, we approximate the series expansions in (40) and (41) by the leading terms,

$$
\begin{aligned}
& I_{0}(x)=1+\frac{x^{2}}{4}+O\left(x^{3}\right), \\
& I_{1}(x)=\frac{x}{2}+\frac{x^{3}}{16}+O\left(x^{4}\right), \\
& I_{2}(x)=\frac{x^{2}}{8}+O\left(x^{3}\right), \\
& K_{1}(x)=\frac{1}{x}+\frac{x}{2} \log \left(\frac{x}{2}\right)+\frac{2 \gamma-1}{4} x+O\left(x^{2}\right),
\end{aligned}
$$

where $\gamma(=0.5772 \cdots)$ is Euler's constant. By substituting (45), (46), (47), and (48) into (44), we obtain the following expression: 


$$
\begin{aligned}
& \dot{g}_{j k}=\kappa \frac{r_{j}}{r_{k}} \frac{r_{j}^{2}+4 \gamma r_{k}^{2}}{8+\kappa^{2} r_{k}^{2}}+\frac{4 \kappa r_{j} r_{k}}{8+\kappa^{2} r_{k}^{2}} \log \left(\frac{\kappa r_{k}}{2}\right)+O\left(\kappa^{2}\right) \\
& \underset{\kappa \rightarrow 0}{\longrightarrow} 0 .
\end{aligned}
$$

For the same reason, $\lim _{\kappa \rightarrow 0} \dot{g}_{j k}=0$ for $r_{j}>r_{k}$. From (38), it follows that $\lim _{\kappa \rightarrow 0} \dot{g}_{j k}=0$ for any $j$ and $k$, which implies that $\lim _{\kappa \rightarrow 0} \dot{M}_{j k}=0$ for any $j$ and $k$. From (37), we thus obtain

$$
\left[\frac{\partial D(\tilde{\lambda}, \kappa)}{\partial \kappa}\right]_{\kappa=0}=0 .
$$

\section{Appendix D: Derivation of Eq. (29)}

Because $\dot{M}_{j k}^{(0)}=\left[\dot{M}_{j k}\right]_{\kappa=0}=0$ (see Appendix C), the second partial derivative of $D(\tilde{\lambda}, \kappa)$ in (36) with respect to $\kappa$ becomes, for $\kappa \rightarrow 0$,

$$
\ddot{D}^{(0)}=\frac{1}{(N-1) !} \epsilon_{j_{1} j_{2} \cdots j_{N}} \epsilon_{k_{1} k_{2} \cdots k_{N}} \ddot{M}_{j_{1} k_{1}}^{(0)} M_{j_{2} k_{2}}^{(0)} \cdots M_{j_{N} k_{N}}^{(0)},
$$

where ()$^{(0)}$ denotes the value of () at $\kappa=0$. From (15) and $g_{j k}^{(0)}=\frac{1}{2} \min \left(\frac{r_{j}}{r_{k}}, \frac{r_{k}}{r_{j}}\right)$, the first two $N \times 1$ column vectors $M_{j_{1} 1}^{(0)}$ and $M_{j_{12}}^{(0)}\left(j_{1}=1,2, \ldots, N\right)$ become

$$
\begin{gathered}
{\left[\begin{array}{c}
M_{11}^{(0)} \\
M_{21}^{(0)} \\
\vdots \\
M_{N 1}^{(0)}
\end{array}\right]=\left[\begin{array}{c}
\frac{1}{2} \Delta Q_{1}+\tilde{\lambda}-\Omega_{1} \\
\frac{r_{1}}{2 r_{2}} \Delta Q_{2} \\
\vdots \\
\frac{r_{1}}{2 r_{N}} \Delta Q_{N}
\end{array}\right]} \\
\text { and }\left[\begin{array}{c}
M_{12}^{(0)} \\
M_{22}^{(0)} \\
\vdots \\
M_{N 2}^{(0)}
\end{array}\right]=\left[\begin{array}{c}
\frac{r_{1}}{2 r_{2}} \Delta Q_{2} \\
\frac{1}{2} \Delta Q_{2}+\tilde{\lambda}-\Omega_{2} \\
\vdots \\
\frac{r_{2}}{2 r_{N}} \Delta Q_{N}
\end{array}\right] .
\end{gathered}
$$

By substituting $\tilde{\lambda}=\Omega_{2}$ into (52), and by using $\Omega_{2}-$ $\Omega_{1}=-\frac{\Delta Q_{1}}{2}+\frac{r_{1}^{2}}{2 r_{2}^{2}} \Delta Q_{1}$ from (16), we see that they are parallel to each other:

$$
M_{j_{1} 1}^{(0)}=\frac{r_{1}}{r_{2}} M_{j_{1} 2}^{(0)}\left(j_{1}=1,2, \ldots, N\right) .
$$

Here, the terms in (51), which include both $M_{j_{1} 1}^{(0)}$ and $M_{j_{1} 2}^{(0)}$, disappear, and only the terms including $\ddot{M}_{j_{1} 1}^{(0)}$ or $\ddot{M}_{j_{12}}^{(0)}$ survive:

$$
\begin{aligned}
\ddot{D}^{(0)}= & \frac{1}{(N-1) !} \epsilon_{j_{1} j_{2} \cdots j_{N}} \epsilon_{1 k_{2} \cdots k_{N}} \ddot{M}_{j_{1} 1}^{(0)} M_{j_{2} k_{2}}^{(0)} \cdots M_{j_{N} k_{N}}^{(0)} \\
& +\frac{1}{(N-1) !} \epsilon_{j_{1} j_{2} \cdots j_{N}} \epsilon_{2 k_{2} \cdots k_{N}} \ddot{M}_{j_{1} 2}^{(0)} M_{j_{2} k_{2}}^{(0)} \cdots M_{j_{N} k_{N}}^{(0)} .
\end{aligned}
$$

The first term on the right-hand side (RHS) of this equation includes $M_{j_{1} 2}^{(0)}$, and the second one includes $M_{j_{1} 1}^{(0)}$. By substituting $M_{j_{1} 1}^{(0)}=\frac{r_{1}}{r_{2}} M_{j_{1} 2}^{(0)}$ into the second term on the RHS of the above equation, and by rewriting $\epsilon_{2 k_{2} \cdots 1 \cdots k_{N}}=-\epsilon_{1 k_{2} \cdots 2 \cdots k_{N}}$, we find that the second derivative $\ddot{D}^{(0)}$ in (15) reduces to

$$
\ddot{D}^{(0)}=\frac{1}{(N-1) !} \epsilon_{j_{1} j_{2} \cdots j_{N}} \epsilon_{1 k_{2} \cdots k_{N}} M_{j_{1}}^{*} M_{j_{2} k_{2}}^{(0)} \cdots M_{j_{N} k_{N}}^{(0)}
$$

where $M_{j_{1}}^{*}=\ddot{M}_{j_{1} 1}^{(0)}-\frac{r_{1}}{r_{2}} \ddot{M}_{j_{1} 2}^{(0)}$. From (15) and $g_{j k}^{(0)}=$ $\frac{1}{2} \min \left(\frac{r_{j}}{r_{k}}, \frac{r_{k}}{r_{j}}\right)$, the first two $1 \times(N-1)$ row vectors $M_{1 k_{1}}^{(0)}$ and $M_{2 k_{1}}^{(0)}\left(k_{1}=2,3, \ldots, N\right)$ become

$$
\begin{aligned}
& {\left[\begin{array}{llll}
M_{12}^{(0)} & M_{13}^{(0)} & \ldots & M_{1 N}^{(0)}
\end{array}\right]} \\
& =\left[\begin{array}{llll}
\frac{r_{1}}{2 r_{2}} \Delta Q_{1} & \frac{r_{1}}{2 r_{3}} \Delta Q_{1} & \cdots & \frac{r_{1}}{2 r_{N}} \Delta Q_{1}
\end{array}\right], \\
& {\left[\begin{array}{lllll}
M_{22}^{(0)} & M_{23}^{(0)} & \cdots & M_{2 N}^{(0)}
\end{array}\right]} \\
& =\left[\begin{array}{llll}
\frac{1}{2} \Delta Q_{2}+\tilde{\lambda}-\Omega_{2} & \frac{r_{2}}{2 r_{3}} \Delta Q_{2} & \cdots & \frac{r_{2}}{2 r_{N}} \Delta Q_{2}
\end{array}\right] .
\end{aligned}
$$

By substituting $\tilde{\lambda}=\Omega_{2}$ and using (16), we see that they are parallel to each other: $M_{1 k_{1}}^{(0)}=\frac{\Delta Q_{1} r_{1}}{\Delta Q_{2} r_{2}} M_{2 k_{1}}^{(0)}\left(k_{1}\right.$ $=2, \ldots, N)$. Therefore, the terms in (53) that include both $M_{1 k_{1}}^{(0)}$ and $M_{2 k_{1}}^{(0)}\left(k_{1}=2, \ldots, N\right)$ disappear, and only the terms including $M_{1}^{*}$ or $M_{2}^{*}$ survive:

$$
\begin{aligned}
\ddot{D}^{(0)}= & \frac{1}{(N-1) !} \epsilon_{1 j_{2} \cdots j_{N}} \epsilon_{1 k_{2} \cdots k_{N}} M_{1}^{*} M_{j_{2} k_{2}}^{(0)} \cdots M_{j_{N} k_{N}}^{(0)} \\
& +\frac{1}{(N-1) !} \epsilon_{2 j_{2} \cdots j_{N}} \epsilon_{1 k_{2} \cdots k_{N}} M_{2}^{*} M_{j_{2} k_{2}}^{(0)} \cdots M_{j_{N} k_{N}}^{(0)} .
\end{aligned}
$$

The first term on the RHS of the above equation in- 
cludes $M_{2 k_{2}}^{(0)}$, and the second includes $M_{1 k_{2}}^{(0)}$. By substituting $M_{1 k_{1}}^{(0)}=\frac{\Delta Q_{1} r_{1}}{\Delta Q_{2} r_{2}} M_{2 k_{1}}^{(0)}\left(k_{1}=2, \ldots, N\right)$ into the second term on the RHS of the above equation, and by rewriting $\epsilon_{2 j_{2} \cdots 1 \cdots j_{N} . .}=-\epsilon_{1 j_{2} \cdots 2 \cdots j_{N}}$, we can reduce the second derivative $\ddot{D}^{(0)}$ in (53) further to

$$
\begin{aligned}
\ddot{D}^{(0)}= & \frac{1}{(N-1) !} \epsilon_{1 j_{2} \cdots j_{N}} \epsilon_{1 k_{2} \cdots k_{N}} \\
& \times\left(M_{1}^{*}-\frac{\Delta Q_{1} r_{1}}{\Delta Q_{2} r_{2}} M_{2}^{*}\right) M_{j_{2} k_{2}}^{(0)} \cdots M_{j_{N} k_{N}}^{(0)} .
\end{aligned}
$$

From (15) and $g_{j k}^{(0)}=\frac{1}{2} \min \left(\frac{r_{j}}{r_{k}}, \frac{r_{k}}{r_{j}}\right)$, the first two $1 \times$ $(N-2)$ row vectors $M_{2 k_{3}}^{(0)}$ and $M_{3 k_{3}}^{(0)}\left(k_{3}=3,4, \ldots, N\right)$ then become

$$
\begin{aligned}
& {\left[\begin{array}{llll}
M_{23}^{(0)} & M_{24}^{(0)} & \ldots & M_{2 N}^{(0)}
\end{array}\right]} \\
& =\left[\begin{array}{llll}
\frac{r_{2}}{2 r_{3}} \Delta Q_{2} & \frac{r_{2}}{2 r_{4}} \Delta Q_{2} & \cdots & \frac{r_{2}}{2 r_{N}} \Delta Q_{2}
\end{array}\right], \\
& {\left[\begin{array}{lllll}
M_{33}^{(0)} & M_{34}^{(0)} & \ldots & M_{3 N}^{(0)}
\end{array}\right]} \\
& =\left[\begin{array}{lllll}
\frac{1}{2} \Delta Q_{3}+\tilde{\lambda}-\Omega_{3} & \frac{r_{3}}{2 r_{4}} \Delta Q_{3} & \ldots & \frac{r_{3}}{2 r_{N}} \Delta Q_{3}
\end{array}\right] .
\end{aligned}
$$

By substituting $\tilde{\lambda}=\Omega_{2}=\Omega_{3}$, we see that they are parallel to each other; that is, $M_{2 k_{3}}^{(0)}=\frac{\Delta Q_{2} r_{2}}{\Delta Q_{3} r_{3}} M_{3 k_{3}}^{(0)}\left(k_{3}=\right.$ $3,4, \ldots, N)$. Therefore, the terms in (54) that include both $M_{2 k_{3}}^{(0)}$ and $M_{3 k_{3}}^{(0)}$ disappear, and only the terms including $M_{22}^{(0)}$ or $M_{32}^{(0)}$ survive:

$$
\begin{aligned}
\ddot{D}^{(0)}= & \left(M_{1}^{*}-\frac{\Delta Q_{1} r_{1}}{\Delta Q_{2} r_{2}} M_{2}^{*}\right) \frac{1}{(N-2) !} \\
& \times\left(\epsilon_{12 j_{3} \cdots j_{N}} \epsilon_{12 k_{3} \cdots k_{N}} M_{22}^{(0)} M_{j_{3} k_{3}}^{(0)} \cdots M_{j_{N} k_{N}}^{(0)}\right. \\
& \left.\quad+\epsilon_{13 j_{3} \cdots j_{N}} \epsilon_{12 k_{3} \cdots k_{N}} M_{32}^{(0)} M_{j_{3} k_{3}}^{(0)} \cdots M_{j_{N} k_{N}}^{(0)}\right) \\
= & \left(M_{1}^{*}-\frac{\Delta Q_{1} r_{1}}{\Delta Q_{2} r_{2}} M_{2}^{*}\right)\left(M_{22}^{(0)}-\frac{\Delta Q_{2} r_{2}}{\Delta Q_{3} r_{3}} M_{32}^{(0)}\right) \\
& \times \frac{1}{(N-2) !} \epsilon_{12 j_{3} \cdots j_{N}} \epsilon_{12 k_{3} \cdots k_{N}} M_{j_{3} k_{3}}^{(0)} \cdots M_{j_{N} k_{N}}^{(0)} .
\end{aligned}
$$

By substituting (15) into the above equation, we obtain the following expression:

$$
\begin{aligned}
\ddot{D}^{(0)}= & \frac{\Delta Q_{1} \Delta Q_{2}}{2}\left\{\left(\ddot{g}_{11}-\frac{r_{1}}{r_{2}} \ddot{g}_{12}\right)-\frac{r_{1}}{r_{2}}\left(\ddot{g}_{21}-\frac{r_{1}}{r_{2}} \ddot{g}_{22}\right)\right\} \\
& \times\left(1-\frac{r_{2}^{2}}{r_{3}^{2}}\right) \frac{1}{(N-2) !} \epsilon_{12 j_{3} \cdots j_{N}} \epsilon_{12 k_{3} \cdots k_{N}} M_{j_{3} k_{3}}^{(0)} \cdots M_{j_{N} k_{N}}^{(0)}
\end{aligned}
$$

Here, $\frac{1}{(N-2) !} \epsilon_{12 j_{3} \cdots j_{N}} \epsilon_{12 k_{3} \cdots k_{N}} M_{j_{3} k_{3}}^{(0)} \cdots M_{j_{N} k_{N}}^{(0)}$ is the determinant of $M=\tilde{\lambda} E-A$ for $\kappa=0$ in the absence of $\Delta Q_{1}$ and $\Delta Q_{2}$. We consider the degenerate case $\tilde{\lambda}=\Omega_{2}$ $=\Omega_{3}$, for which $\Delta Q_{1} r_{1}^{2}+\Delta Q_{2} r_{2}^{2}=0$. Then, from (16), the basic angular velocity $\Omega_{j}(j \geq 3)$ does not depend on $\Delta Q_{1}$ or $\Delta Q_{2}$. Therefore, by the same reasoning as that used to derive $D(\lambda, 0)=\prod_{j=2}^{N+1}\left(\lambda-\Omega_{j}\right)$ (see Appen$\operatorname{dix} \mathrm{A})$, we can derive the following expression:

$$
\begin{aligned}
& \frac{1}{(N-2) !} \epsilon_{12 j_{3} \cdots j_{N}} \epsilon_{12 k_{3} \cdots k_{N}} M_{j_{3} k_{3}}^{(0)} \cdots M_{j_{N} k_{N}}^{(0)} \\
& =\prod_{j=4}^{N+1}\left(\tilde{\lambda}-\Omega_{j}\right)=\prod_{j=4}^{N+1}\left(\Omega_{2}-\Omega_{j}\right) .
\end{aligned}
$$

By substituting (57) into (56), we obtain the following expression:

$$
\ddot{D}^{(0)}=\frac{\Delta Q_{1} \Delta Q_{2}}{2}\left(1-\frac{r_{2}^{2}}{r_{3}^{2}}\right) F \prod_{j=4}^{N+1}\left(\Omega_{2}-\Omega_{j}\right),
$$

where

$$
\begin{aligned}
F & =\left(\ddot{g}_{11}-\frac{r_{1}}{r_{2}} \ddot{g}_{12}\right)-\frac{r_{1}}{r_{2}}\left(\ddot{g}_{21}-\frac{r_{1}}{r_{2}} \ddot{g}_{22}\right) \\
& =\ddot{g}_{11}+\frac{r_{1}^{2}}{r_{2}^{2}} \ddot{g}_{22}-2 \frac{r_{1}}{r_{2}} \ddot{g}_{12} .
\end{aligned}
$$

By using the following equations for the modified Bessel functions,

$$
\begin{aligned}
& \frac{d^{2} I_{n}}{d x^{2}}=\left(1+\frac{n^{2}}{x^{2}}\right) I_{n}(x)-\frac{1}{x} \frac{d I_{n}}{d x}, \\
& \frac{d^{2} K_{n}}{d x^{2}}=\left(1+\frac{n^{2}}{x^{2}}\right) K_{n}(x)-\frac{1}{x} \frac{d K_{n}}{d x},
\end{aligned}
$$

we can express the second derivatives $\ddot{g}_{j k}$ of $g_{j k}=$ $I_{1}\left(\kappa r_{j}\right) K_{1}\left(\kappa r_{k}\right)(j \leq k)$ with respect to $\kappa$ as follows:

$$
\begin{aligned}
\ddot{g}_{j k}= & \left(r_{j}^{2}+r_{k}^{2}+\frac{2}{\kappa^{2}}\right) I_{1}\left(\kappa r_{j}\right) K_{1}\left(\kappa r_{k}\right) \\
& -\frac{r_{j}}{\kappa} I_{1}^{\prime}\left(\kappa r_{j}\right) K_{1}\left(\kappa r_{k}\right)-\frac{r_{k}}{\kappa} I_{1}\left(\kappa r_{j}\right) K_{1}^{\prime}\left(\kappa r_{k}\right) \\
& +2 r_{j} r_{k} I_{1}^{\prime}\left(\kappa r_{j}\right) K_{1}^{\prime}\left(\kappa r_{k}\right) \\
= & \left(r_{j}^{2}+r_{k}^{2}+\frac{2}{\kappa^{2}}\right) I_{1}\left(\kappa r_{j}\right) K_{1}\left(\kappa r_{k}\right)-\frac{1}{\kappa} \dot{g}_{j k} \\
& +2 r_{j} r_{k} I_{1}^{\prime}\left(\kappa r_{j}\right) K_{1}^{\prime}\left(\kappa r_{k}\right) .
\end{aligned}
$$


In the vicinity of $\kappa=0$, by substituting (45), (46), (47), (48), and (49) into the first, second, and third terms on the RHS of (62), respectively, we obtain the following expressions:

$$
\begin{aligned}
& \text { the first term }= \frac{r_{j}}{r_{k}}\left(\frac{5}{8} r_{j}^{2}+\frac{2 \gamma+1}{4} r_{k}^{2}+\frac{1}{\kappa^{2}}\right) \\
&+ \frac{r_{j} r_{k}}{2} \log \left(\frac{\kappa r_{k}}{2}\right)+O\left(\kappa^{2}\right), \\
& \text { the second term }=-\frac{r_{j}}{r_{k}} \frac{r_{j}^{2}+4 \gamma r_{k}^{2}}{8+\kappa^{2} r_{k}^{2}}-\frac{4 r_{j} r_{k}}{8+\kappa^{2} r_{k}^{2}} \log \left(\frac{\kappa r_{k}}{2}\right) \\
&+O\left(\kappa^{2}\right), \\
& \text { the third term }= 2 r_{j} r_{k} \frac{I_{0}\left(\kappa r_{j}\right)+I_{2}\left(\kappa r_{j}\right)}{2} \frac{1}{I_{1}\left(\kappa r_{k}\right)} \\
& \times\left\{\frac{I_{0}\left(\kappa r_{k}\right)+I_{2}\left(\kappa r_{k}\right)}{2} K_{1}\left(\kappa r_{k}\right)-\frac{1}{\kappa r_{k}}\right\} \\
&= \frac{r_{j}}{r_{k}} \frac{1}{8+\kappa^{2} r_{k}^{2}}\left\{-\frac{8}{\kappa^{2}}-3 r_{j}^{2}+(4 \gamma+1) r_{k}^{2}\right. \\
&\left.+4 r_{k}^{2} \log \left(\frac{\kappa r_{k}}{2}\right)\right\}+O\left(\kappa^{2}\right) .
\end{aligned}
$$

From (63), (64), and (65), (62) becomes

$$
\begin{aligned}
\ddot{g}_{j k}= & \frac{r_{j}}{r_{k}}\left(\frac{5}{8} r_{j}^{2}+\frac{2 \gamma+1}{4} r_{k}^{2}+\frac{1}{\kappa^{2}}\right)+\frac{r_{j} r_{k}}{2} \log \left(\frac{\kappa r_{k}}{2}\right) \\
& -\frac{r_{j}}{r_{k}} \frac{r_{j}^{2}+4 \gamma r_{k}^{2}}{8+\kappa^{2} r_{k}^{2}}-\frac{4 r_{j} r_{k}}{8+\kappa^{2} r_{k}^{2}} \log \left(\frac{\kappa r_{k}}{2}\right) \\
& +\frac{r_{j}}{r_{k}} \frac{1}{8+\kappa^{2} r_{k}^{2}}\left\{-\frac{8}{\kappa^{2}}-3 r_{j}^{2}+(4 \gamma+1) r_{k}^{2}\right. \\
= & \left.r_{j} r_{k}\left(\frac{2 \gamma+1}{4}+\frac{2}{8+\kappa^{2} r_{k}^{2}}\right)+\frac{r_{j}^{3}}{r_{k}}\left(\frac{5}{8}-\frac{4}{8+\kappa_{k}^{2} r_{k}^{2}}\right)\right\}+O\left(\kappa^{2}\right) \\
& +\frac{r_{j} r_{k}}{2} \log \left(\frac{\kappa r_{k}}{2}\right)+O\left(\kappa^{2}\right) .
\end{aligned}
$$

By substituting (66) into (59) and taking the limit $\kappa \rightarrow$ 0 , we obtain the following expression:

$$
\begin{aligned}
& F=\ddot{g}_{11}+\frac{r_{1}^{2}}{r_{2}^{2}} \ddot{g}_{22}-2 \frac{r_{1}}{r_{2}} \ddot{g}_{12} \\
& =r_{1}^{2}\left\{\frac{2 \gamma+1}{4}+\frac{2}{8+\kappa^{2} r_{1}^{2}}+\frac{5}{8}-\frac{4}{8+\kappa^{2} r_{1}^{2}}+\frac{2 \gamma+1}{4}\right. \\
& +\frac{2}{8+\kappa^{2} r_{2}^{2}}+\frac{5}{8}-\frac{4}{8+\kappa^{2} r_{2}^{2}} \\
& -2\left(\frac{2 \gamma+1}{4}+\frac{2}{8+\kappa^{2} r_{2}^{2}}\right)-2 \frac{r_{1}^{2}}{r_{2}^{2}}\left(\frac{5}{8}-\frac{4}{8+\kappa^{2} r_{2}^{2}}\right) \\
& \left.+\frac{1}{2} \log \left(\frac{r_{1}}{r_{2}}\right)\right\}+O\left(\kappa^{2}\right) \\
& \underset{\kappa \rightarrow 0}{\longrightarrow} \frac{r_{1}^{2}}{2}\left\{\frac{1-\frac{r_{1}^{2}}{r_{2}^{2}}}{2}+\log \left(\frac{r_{1}}{r_{2}}\right)\right\} \text {. }
\end{aligned}
$$

Substituting (67) into (58), we finally obtain

$$
\begin{aligned}
\ddot{D}^{(0)}= & {\left[\frac{\partial^{2} D(\tilde{\lambda}, \kappa)}{\partial \kappa^{2}}\right]_{\kappa=0} } \\
= & \frac{\Delta Q_{1} \Delta Q_{2}}{8} r_{1}^{2}\left(1-\frac{r_{2}^{2}}{r_{3}^{2}}\right)\left\{1-\frac{r_{1}^{2}}{r_{2}^{2}}+2 \log \left(\frac{r_{1}}{r_{2}}\right)\right\} \\
& \times \prod_{j=4}^{N+1}\left(\Omega_{2}-\Omega_{j}\right) .
\end{aligned}
$$

\section{Reference}

Chen, Y., and M. K. Yau, 2001: Spiral bands in a simulated hurricane. Part I: Vortex Rossby wave verification. $J$. Atmos. Sci., 58, 2128-2145.

Flierl, G. R., 1988: On the stability of geostrophic vortices. $J$. Fluid. Mech., 197, 349-388.

Gao, C., and P. Zhu, 2016: Vortex Rossby wave propagation in baroclinic tropical cyclone-like vortices. Geophys. Res. Lett., 43, 12578-12589.

Hoskins, B. J., and F. P. Bretherton, 1972: Atmospheric frontogenesis models: Mathematical formulation and solution. J. Atmos. Sci., 29, 11-37.

Hoskins, B. J., M. E. McIntyre, and A. W. Robertson, 1985: On the use and significance of isentropic potential vorticity maps. Quart. J. Roy. Meteor. Soc., 111, 877946.

Ito, T., and H. Kanehisa, 2013: Analytical solutions of vortex Rossby waves in a discrete barotropic model. $J$. Meteor. Soc. Japan, 91, 775-788.

Montgomery, M. T., and R. J. Kallenbach, 1997: A theory for vortex Rossby-waves and its application to spiral bands and intensity changes in hurricanes. Quart. $J$. Roy. Meteor. Soc., 123, 435-465.

Nishimoto, S., and H. Kanehisa, 2018: Analytical solutions of vortex Rossby waves associated with vortex resil- 
iency of tropical cyclones. J. Meteor. Soc. Japan, 96, $5-24$.

Nolan, D. S., and M. T. Montgomery, 2000: The algebraic growth of wavenumber one disturbances in hurricanelike vortices. J. Atmos. Sci., 57, 3514-3538.

Peng, M. S., J. Peng, T. Li, and E. Hendricks, 2014: Effect of baroclinicity on vortex axisymmetrization. Part I: Barotropic basic vortex. Adv. Atmos. Sci., 31, 12561266.

Peng, J., M. S. Peng, T. Li, and E. Hendricks, 2014: Effect of baroclinicity on vortex axisymmetrization. Part II: Baroclinic basic vortex. Adv. Atmos. Sci., 31, 12671278.

Reasor, P. D., and M. T. Montgomery, 2001: Three-dimensional alignment and corotation of weak, TC-like vortices via linear vortex Rossby waves. J. Atmos. Sci., 58, 2306-2330.

Reznik, G. M., and W. K. Dewar, 1994: An analytical theory of distributed axisymmetric barotropic vortices on the $\beta$-plane. J. Fluid. Mech., 269, 301-321.

Schubert, W. H., M. T. Montgomery, R. K. Taft, T. A. Guinn, S. R. Fulton, J. P. Kossin, and J. P. Edwards, 1999: Polygonal eyewalls, asymmetric eye contraction, and potential vorticity mixing in hurricanes. J. Atmos. Sci., 56, 1197-1223.

Smith, R. A., and M. N. Rosenbluth, 1990: Algebraic instability of hollow electron columns and cylindrical vortices. Phys. Rev. Lett., 64, 649-652.

Terwey, W. D., and M. T. Montgomery, 2002: Wavenumber-2 and wavenumber- $m$ vortex Rossby wave instabilities in a generalized three-region model. J. Atmos. Sci., 59, 2421-2427.

Wang, Y., 2002: Vortex Rossby waves in a numerically simulated tropical cyclone. Part I: Overall structure, potential vorticity, and kinetic energy budgets. $J$. Atmos. Sci., 59, 1213-1238. 